\title{
Cardiovascular MRI in Detection and Measurement of Aortic Atheroma in Stroke/TIA patients
}

Theodore Faber ${ }^{1}$, Ashley Rippy ${ }^{1}$, W Brian Hyslop ${ }^{2}$, Alan Hinderliter ${ }^{2}$ and Souvik Sen ${ }^{1 *}$

${ }^{1}$ University of South Carolina School of Medicine, Department of Neurology, Columbia, South Carolina, USA

${ }^{2}$ University of North Carolina School of Medicine, Department of Radiology and Cardiology, Chapel Hill, North Carolina, USA

\begin{abstract}
Background: Aortic Atheroma $(\mathrm{A} A \mathrm{~A})$ is an independent risk factor for new and recurrent stroke. AoA ulceration and mobility are associated with an increased risk for brain embolism. Transesophageal echocardiography (TEE) is the gold standard for detection and measurement of AoA in stroke/TIA patients. Cardiovascular MRI (cMRI) could be an alternative, non-invasive imaging modality for stroke/TIA patients. The objective of this study was to assess the accuracy and correlation of AoA detected and measured by cMRI versus TEE in patients with recent stroke/TIA
\end{abstract}

Methods and results: Twenty-two stroke/TIA patients undergoing TEE as a part of their stroke workup consented to a protocol-mandated cMRI performed on a $1.5 \mathrm{~T}$ magnet. The protocol included an axial non-breathhold EKGgated dual-echo spin echo MRI of the thoracic aorta (TR/TE1/TE2=900/29/69) and a contrast-enhanced breathhold $3 \mathrm{D}$ gradient-echo image of the thorax (flip/TR/TE=12/4.0/1.71). Maximum plaque thickness, ulceration $(\geq 2 \mathrm{~mm})$ and mobility of AoA were assessed in the proximal (ascending and proximal arch) and distal (distal arch and descending) segments of thoracic aorta by a cardiologist to interpret the TEE and a radiologist to interpret the cMRI. There was good correlation between $c M R I$ and TEE in measurement of plaque thickness in the proximal segments $(R=0.73, p<0.0001)$ and the distal segments $(R=0.81, p<0.0001)$ of the aortic arch $(A A)$. cMRI had a high degree of accuracy in detecting measurable AoA ( $\geq 1 \mathrm{~mm}$ ) in the proximal segments (sensitivity $90 \%$, specificity $100 \%$ ), as well as the distal segments (sensitivity $67 \%$, specificity $100 \%$ ). cMRI also had a high degree of accuracy in detecting significant AoA ( $\geq 4 \mathrm{~mm})$ in proximal segments (sensitivity $71 \%$, specificity $93 \%$ ), as well as distal segments (sensitivity $71 \%$, specificity $100 \%$ ).

Conclusion: The study showed a high degree of accuracy and correlation of AoA detected and measured by cMRI as compared to TEE in patients with recent stroke/TIA. This technique has limitations in detection of AoA ulceration, and protocols assessing AoA mobility need to be developed.

Keywords: Cardiovascular MRI; Transesophogeal echocardiogram; Aortic atheroma

\section{Introduction}

By any measure, the impact of stroke in the developed world is substantial. In the U.S., stroke is the fourth leading cause of death and a primary cause of significant disability [1]. It is essential to identify the source of stroke for appropriate treatment and secondary prevention [2]. Cryptogenic stroke, i.e. stroke without identifiable cause, may account for $30-40 \%$ of all stroke [3-6] and an increasingly recognized source of embolic stroke appears to be atheroma of the thoracic aorta, second only to atrial fibrillation in terms of risk [7-11]. Proximal aortic arch (PAA) atheroma plaques are seen in $16-20 \%$ of stroke/TIA patients [7]. Distal aortic arch (DAA) atheromatous plaque burden is a predictor of coronary disease [12], visceral thromboembolism, and mortality [13]. The disease burden in proximal DAA has also been demonstrated to be a frequent source of stroke via retrograde flow by Harloff et al. in 2010 [14]. Transesophageal echocardiography (TEE) is routinely requested in patients with cryptogenic stroke in order to identify a potential embolic source; however, it is moderately invasive and not tolerated by all patients. Moreover, the DAA is sometimes difficult to visualize due to airway obscuration [15]. Magnetic resonance imaging (MRI) is routinely used to investigate embolic potential from atheromatous disease in carotid arteries. This study proposes to analyze whether MRI can be useful to investigate embolic potential from proximal and distal aortic arch.

In this study, cardiovascular MRI (cMRI) of the aortic arch (AA) was used to visualize the AA and identify aortic atheroma (AoA) in cryptogenic ischemic stroke and transient ischemic attack (TIA) patients. Correlation with TEE in 22 consecutive patients with cryptogenic ischemic stroke and TIA was made in order to assess whether cMRI was able to identify AoA in these patients and to compare the characteristics of lesions identified by cMRI to those revealed by TEE.

\section{Methods and Materials}

\section{Subjects}

With institutional review board (UNC Biomedical IRB) approval, twenty-two ischemic stroke/TIA patients (mean age 68 years, 11 males, 17 white, 3 African-American and 2 others) undergoing TEE for assessment of possible cardiac source of embolism at UNC Chapel Hill served as subjects for the study. All patients experienced a cryptogenic stroke as defined by TOAST criteria [16]. To be included in the study, patients had to be age 18 or above, with stroke or TIA in the last 90 days, AA atheroma detected and measured on a clinically indicated TEE, and the ability to consent. Key exclusion criteria included the inability to cooperate with the performance of the MRI test due to

*Corresponding author: Souvik Sen, Professor and Chair, Department of Neurology, University of South Carolina School of Medicine, 8 Medical Park, Suite 420 Columbia, South Carolina 29203, USA, Tel: 803-545-6050/6073; Fax: 803 545-6051; E-mail: Souvik.Sen@uscmed.sc.edu

Received October 04, 2013; Accepted October 30, 2013; Published November 06, 2013

Citation: Faber T, Rippy A, Brian Hyslop W, Hinderliter A, Sen S (2013) Cardiovascular MRI in Detection and Measurement of Aortic Atheroma in Stroke/ TIA patients. J Neurol Disord 1: 139. doi:10.4172/2329-6895.1000139

Copyright: (c) 2013 Faber T, et al. This is an open-access article distributed unde the terms of the Creative Commons Attribution License, which permits unrestricted use, distribution, and reproduction in any medium, provided the original author and source are credited. 
size or claustrophobia, prior history of decreased renal function, and irregular heart rate that could limit cMRI. Patients were also excluded if they were unable to undergo MRI scans due to a history of implanted ferromagnetic material or devices.

\section{Transesophageal echocardiography (TEE)}

Multiplanar TEE was performed on each patient. All patients received topical lidocaine pharyngeal anesthetic and adequate conscious sedation was induced by intravenous midazolam and fentanyl. The $5.0 \mathrm{MHz}$ transducer of a Hewlett-Packard model 21364A transesophageal probe was used (Hewlett-Packard, Andover, Massachusetts). A Hewlett-Packard Sonos 1000 system was utilized and digital images were recorded for subsequent analysis. Imaging and quantification of AoA was conducted according to previously described methods [7]. In brief, the proximal and midascending aorta were imaged at a probe depth of $30 \mathrm{~cm}$ with a multiplane angle of $100^{\circ}$ to $150^{\circ}$ to view the vessel in the long axis. The descending thoracic aorta was examined by advancing the probe to the distal esophagus, imaging the aorta in cross section (at $0^{\circ}$ ), and then slowly withdrawing the probe to image the proximal segments. As the transducer reached the aortic arch plaque, the multiplane angle was rotated to between $0^{\circ}$ and $90^{\circ}$ to acquire sequential short-axis views. Digital images were acquired of the diseased areas in each segment of the aorta with annotation of the distance of the transducer from the incisors. None of these patients had fever or TEE evidence of valvular vegetation suggestive of infective endocarditis.

\section{Aortic Arch Plaque Thickness (AAPT) measurement}

AAPT measurements are the maximal intima-media thickness (IMT) and grade of aortic atheroma [9]. In each patient the area of maximum aortic arch plaque was identified and imaged with Doppler turned off. The $2 \mathrm{D}$ images through the plaque were then obtained. A minimum of 2-3 images were captured and recorded for the arch segment of the aorta.

\section{Cardiovascular MRI (cMRI)}

A 1.5 T magnet cMRI was used for all studies. The 3T magnet was used to optimize imaging and further improve resolution. An axial non-breathhold EKG-gated dual echo spin echo MRI of the thoracic aorta $(\mathrm{TR} / \mathrm{TE} 1 / \mathrm{TE} 2=900 / 29 / 69)$ and a contrast enhanced breathhold $3 \mathrm{D}$ gradient-echo image of the thorax (flip/TR/TE=12/4.0/1.71) was performed on each patient. AA was assessed using spin-echo and $3 \mathrm{D}$ images. Plaque thickness was best assessed using the turbo spin-echo images to discriminate between the native aortic wall and plaque. The turbo spin-echo imaging had a repetition rate of three (3 averages). For larger areas of plaque that invaginated into the aortic lumen, contrastenhanced imaging was employed. Plaque thickness was measured using measurements tools on the agfa PACS.

\section{Results}

There was good correlation between cMRI and TEE measurements of plaque thickness in both proximal segments (Pearson's $R=0.726$ and $p$-value $<0.001$ ) and distal segments (Pearson's $R=0.812$ and p-value $<0.001$ ) of the aortic arch (Figures 1 and 2). cMRI detected of proximal segment measurable AA disease $\geq 1 \mathrm{~mm}$ in thickness with $90 \%$ sensitivity and $100 \%$ specificity. In distal segments of the aortic arch, the sensitivity was $67 \%$ and specificity was $100 \%$. cMRI detected severe AoA disease ( $\geq 4 \mathrm{~mm}$ in thickness) in proximal segments of the aortic arch with $71 \%$ sensitivity and $93 \%$ specificity. In the distal aortic arch, the technique was $71 \%$ specific and $100 \%$ sensitive. cMRI was able to identify $89 \%$ of proximal aorta ulcerations and $64 \%$ of distal aorta

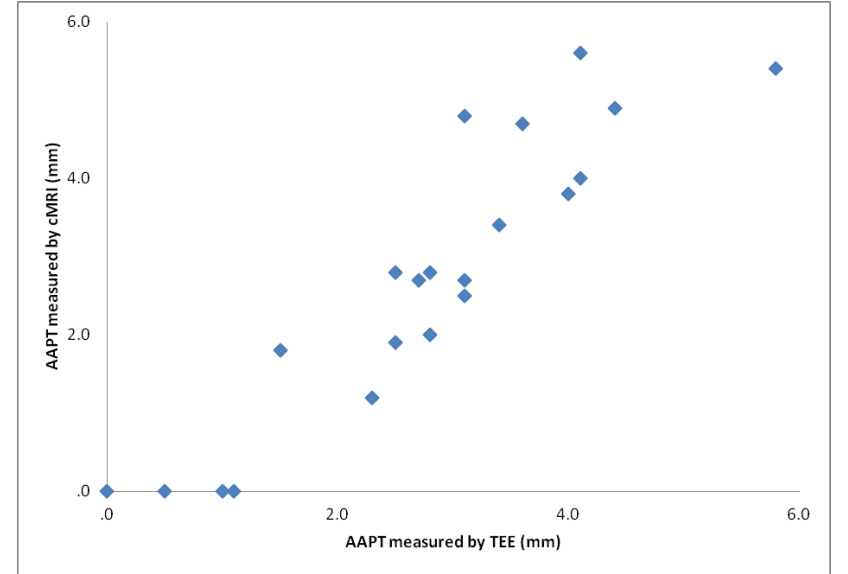

Figure 1: AAPT correlation in proximal segments.

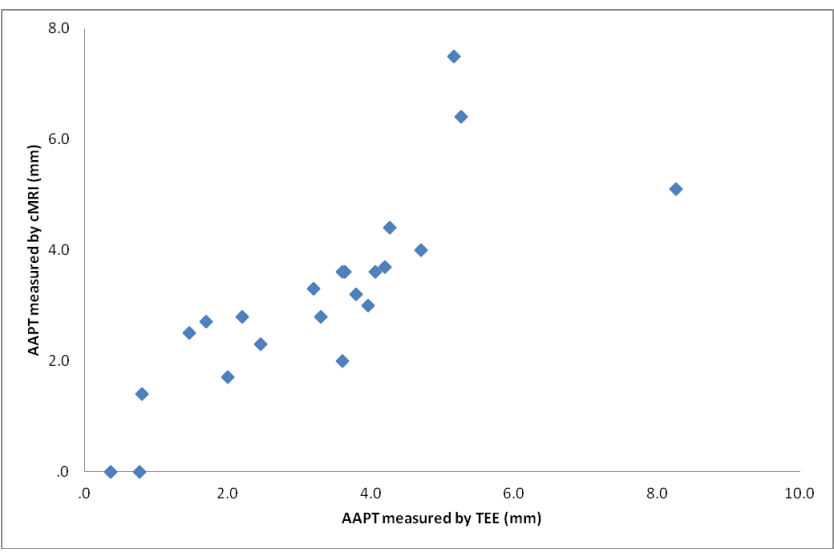

Figure 2: AAPT correlation in distal segments.

ulcerations (Figure 3). The mobility of plaques could not be assessed with this protocol.

\section{Discussion}

It has been shown that aortic atheromatous (AoA) disease may be found in $16-20 \%$ of patients with transient ischemic attack (TIA) or stroke and a number of prospective studies have confirmed a strong association between stroke and AoA plaques [7,8,17-20]. Sen et al. [21] found a high rate of progression of aortic atheroma in $28 \%$ of ischemic stroke/TIA patients using transesophageal echocardiography (TEE), which is considered the gold standard for detection of AoA plaque in this setting [22]. TEE is widely available, produces images of greater resolution than transthoracic echocardiography (TTE) and has high inter-reader reproducibility [23,24]. However, TEE has significant limitations. It is semi-invasive, requires conscious sedation, and carries risk of damage to the esophagus, albeit low [24]. Patient tolerability may be a problem [25]; a relatively large number of individuals refused a follow-up TEE after a 12 month interval in one series [21]. Because of the semi-invasive nature of the study, patients for TEE should be hemodynamically stable. Many patients with stroke have difficulty in swallowing [26] and thus may have difficulty swallowing the transducer. Air artifact and insonation angles can interfere with visualization in proximal $[15,24]$ and distal aortic arch [27]. A failure to reliably assess the aortic arch because of insufficient visualization was noted in one study in about half the patients tested; it was shown that TEE may 

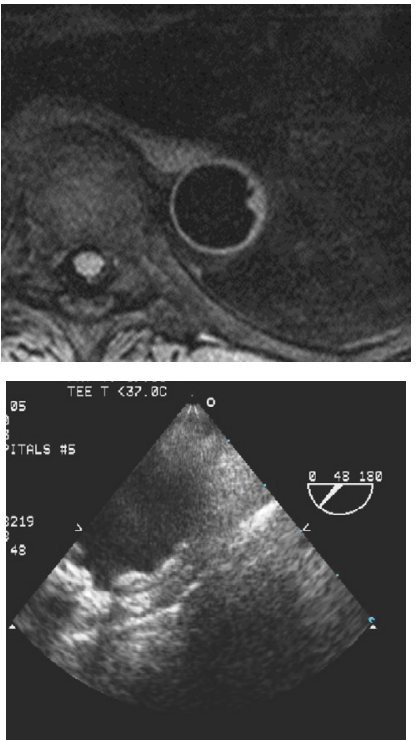

Figure 3: AA ulceration seen on cMRI and TEE.

underestimate AoA disease [27]. Difficulties with plaque thickness measurement and gradation scoring by TEE at precise locations in baseline and follow-up measurements was a limiting factor in a study of progression of AoA disease and risk of stroke [28].

Cardiovascular magnetic resonance imaging (cMRI) of the thoracic aorta has been shown to have unique characteristics which may improve the potential for identifying the source of cryptogenic stroke; about $21 \%$ of a group of patients with stroke of undetermined etiology were found to have AoA disease with embolic potential which was not detected by TEE [27]. The development of new protocols for cMRI to assess atheromatous disease of the ascending and descending aortic arch may help to reduce the numbers of cryptogenic stroke and to help understand the pathophysiology of AoA disease. Analysis of the morphology and potential stabilizing or destabilizing features related to the makeup of the plaque can be identified by cMRI, potentially leading to improved understanding of the processes by which AoA disease progresses [29-32]. Descending aortic arch AoA disease was not recognized as a potential source of stroke $[33,34]$ until it was shown by Harloff et al. [14] using cMRI that retrograde flow with subsequent destabilization of complex atheromatous plaques in the proximal portions of DAA could potentially embolize to all supra-aortic arteries. This finding extended the risk of stroke by retrograde embolization to all brain territories and retina in about one-fourth (9/37) of serial unselected patients with cryptogenic stroke. MRI imaging of associated cardiac structures can be useful in detecting left ventricular thrombus [35-37], left atrial appendage thrombus [38], and patent foramen ovale, although evaluation of the latter requires contrast enhancement $[39,40]$. Reliable and accurate co-registration techniques utilizing cMRI may resolve precision issues [39]. Finally, cMRI may streamline ischemic stroke/TIA evaluation with concomitant imaging of the brain, cerebral and extracerebral vasculature, and cardiac/AA regions with a single examination which may impact overall costs associated with hospitalization [41].

MRI in general and cMRI specifically have limitations. The machines are not portable and represent a substantial investment on the part of the health care system. The presence of hardware including cardiac pacemakers, implanted defibrillators, and cochlear implants which might preclude cMRI is not uncommon in this patient population. Problems with patient size and potential for claustrophobia can be limiting factors. MRI is not performed in patients who are hemodynamically unstable, although this is also a limitation of TEE as noted above. EKG gating techniques may not be able to accommodate very irregular cardiac rhythms [41]. Portions of the cMRI sequence that require a breath-hold may not be feasible for some patients [41]. Finally, plaque mobility could not be assessed using the current protocol, although cine-cMRI [30] and other advanced techniques [42] may help overcome this limitation.

\section{Conclusion}

This study demonstrates a high degree of accuracy and reliability of cMRI of the aortic arch in patients with cryptogenic ischemic stroke/TIA as compared to TEE. To our knowledge, this is the first and largest group of patients with cryptogenic stroke undergoing comparative evaluations of these two imaging techniques. Baher et al. confirmed the effectiveness of the ability of cMRI to reduce the rate of cryptogenic classification in a group of 15 patients (10/15 reclassified as cardioembolic) with cryptogenic stroke in 2012 [25]. Limitations of our study included the modest size of the study and lack of ability to visualize plaque mobility and limited ability to detect ulceration. Continued refinement of cMRI techniques is needed to overcome the technical limitations. Further investigation with greater numbers of subjects with longitudinal analyses of treatment is recommended in order to further understanding of the natural history of AoA disease and to determine optimal treatment paradigms in acute stroke and TIA as well as stroke prevention. Economic analysis of a streamlined MRI approach to the evaluation of cryptogenic ischemic stroke/TIA may be helpful considering the current concerns regarding healthcare expenditures.

\section{Funding}

Dr. Sen is supported by NINDS Grant number 1R01NS062754.

\section{References}

1. Go AS, Mozaffarian D, Roger VL, Benjamin EJ, Berry JD, et al. (2013) Executive summary: heart disease and stroke statistics--2013 update: a report from the American Heart Association. Circulation 127: 143-152.

2. Morris JG, Duffis EJ, Fisher M (2009) Cardiac workup of ischemic stroke: can we improve our diagnostic yield? Stroke 40: 2893-2898.

3. Sacco RL, Ellenberg JH, Mohr JP, Tatemichi TK, Hier DB, et al. (1989) Infarcts of undetermined cause: the NINCDS Stroke Data Bank. Ann Neurol 25: 382 390.

4. Petty GW, Brown RD Jr, Whisnant JP, Sicks JD, O'Fallon WM, et al. (1999) Ischemic stroke subtypes: a population-based study of incidence and risk factors. Stroke 30: 2513-2516.

5. Lee BI, Nam HS, Heo JH, Kim DI; Yonsei Stroke Team (2001) Yonsei Stroke Registry. Analysis of 1,000 patients with acute cerebral infarctions. Cerebrovasc Dis 12: 145-151.

6. Kolominsky-Rabas PL, Weber M, Gefeller O, Neundoerfer B, Heuschmann PU (2001) Epidemiology of ischemic stroke subtypes according to TOAST criteria: incidence, recurrence, and long-term survival in ischemic stroke subtypes: a population-based study. Stroke 32: 2735-2740.

7. Sen S, Wu K, McNamara R, Lima J, Piantadosi S, et al. (2000) Distribution, severity and risk factors for aortic atherosclerosis in cerebral ischemia. Cerebrovasc Dis 10: 102-109.

8. Tunick PA, Rosenzweig BP, Katz ES, Freedberg RS, Perez JL, et al. (1994) High risk for vascular events in patients with protruding aortic atheromas: a prospective study. J Am Coll Cardiol 23: 1085-1090.

9. Amarenco P, Cohen A, Tzourio C, Bertrand B, Hommel M, et al. (1994) Atherosclerotic disease of the aortic arch and the risk of ischemic stroke. $N$ Engl J Med 331: 1474-1479. 
Citation: Faber T, Rippy A, Brian Hyslop W, Hinderliter A, Sen S (2013) Cardiovascular MRI in Detection and Measurement of Aortic Atheroma in Stroke/TIA patients. J Neurol Disord 1: 139. doi:10.4172/2329-6895.1000139

10. Karalis DG, Chandrasekaran K, Victor MF, Ross JJ Jr, Mintz GS (1991) Recognition and embolic potential of intraaortic atherosclerotic debris. J Am Coll Cardiol 17: 73-78.

11. Tunick PA, Perez JL, Kronzon I (1991) Protruding atheromas in the thoracic aorta and systemic embolization. Ann Intern Med 115: 423-427.

12. Fazio GP, Redberg RF, Winslow T, Schiller NB (1993) Transesophagea echocardiographically detected atherosclerotic aortic plaque is a marker for coronary artery disease. J Am Coll Cardiol 21: 144-150.

13. Solberg LA, Strong JP (1983) Risk factors and atherosclerotic lesions. A review of autopsy studies. Arteriosclerosis 3: 187-198.

14. Harloff A, Simon J, Brendecke S, Assefa D, Helbing T, et al. (2010) Complex plaques in the proximal descending aorta: an underestimated embolic source of stroke. Stroke 41: 1145-1150.

15. Konstadt SN, Reich DL, Quintana C, Levy M (1994) The ascending aorta: how much does transesophageal echocardiography see? Anesth Analg 78: 240244

16. Adams HP Jr, Bendixen BH, Kappelle LJ, Biller J, Love BB, et al. (1993) Classification of subtype of acute ischemic stroke. Definitions for use in a multicenter clinical trial. TOAST. Trial of Org 10172 in Acute Stroke Treatment. Stroke 24: 35-41.

17. Zabalgoitia M, Halperin JL, Pearce LA, Blackshear JL, Asinger RW, et al (1998) Transesophageal echocardiographic correlates of clinical risk of thromboembolism in nonvalvular atrial fibrillation. Stroke Prevention in Atrial Fibrillation III Investigators. J Am Coll Cardiol 31: 1622-1666.

18. Mitusch R, Doherty C, Wucherpfennig H, Memmesheimer C, Tepe C, et al. (1997) Vascular events during follow-up in patients with aortic arch atherosclerosis. Stroke 28: 36-39.

19. Ferrari E, Vidal R, Chevallier T, Baudouy M (1999) Atherosclerosis of the thoracic aorta and aortic debris as a marker of poor prognosis: benefit of oral anticoagulants. J Am Coll Cardiol 33: 1317-1322.

20. Fujimoto S, Yasaka M, Otsubo R, Oe H, Nagatsuka K, et al. (2004) Aortic arch atherosclerotic lesions and the recurrence of ischemic stroke. Stroke 35: 1426-1429.

21. Sen S, Hinderliter A, Sen PK, Simmons J, Beck J, et al. (2007) Aortic arch atheroma progression and recurrent vascular events in patients with stroke or transient ischemic attack. Circulation 116: 928-935.

22. Kutz SM, Lee VS, Tunick PA, Krinsky GA, Kronzon I (1999) Atheromas of the thoracic aorta: A comparison of transesophageal echocardiography and breathhold gadolinium-enhanced 3-dimensional magnetic resonance angiography. $J$ Am Soc Echocardiogr 12: 853-858.

23. Harloff A, Handke M, Reinhard M, Geibel A, Hetzel A (2006) Therapeutic strategies after examination by transesophageal echocardiography in 503 patients with ischemic stroke. Stroke 37: 859-864.

24. Thenappan T, Ali Raza J, Movahed A (2008) Aortic atheromas: current concepts and controversies-a review of the literature. Echocardiography 25 : 198-207.

25. Baher A, Mowla A, Volpi JJ, Shah DJ (2012) Cardiac MRI improves identification of etiology of ischemic stroke. J Cardiovasc Magn Reson, 2012. 14 (Suppl1): 79.

26. Martino R, Foley N, Bhogal S, Diamant N, Speechley M, et al. (2005) Dysphagia after stroke: incidence, diagnosis, and pulmonary complications. Stroke 36 2756-2763.

27. Harloff A, Dudler P, Frydrychowicz A, Strecker C, Stroh AL, et al. (2008) Reliability of aortic MRI at 3 Tesla in patients with acute cryptogenic stroke. J Neurol Neurosurg Psychiatry 79: 540-546.
28. Sen S, Oppenheimer SM, Lima J, Cohen B (2002) Risk factors for progression of aortic atheroma in stroke and transient ischemic attack patients. Stroke 33 930-935.

29. Sen S (2009) Aortic arch plaque in stroke. Curr Cardiol Rep 11: 28-35

30. Gottsegen JM, Coplan NL (2008) The atherosclerotic aortic arch: considerations in diagnostic imaging. Prev Cardiol 11: 162-167.

31. Fayad ZA, Nahar T, Fallon JT, Goldman M, Aguinaldo JG, et al. (2000) In vivo magnetic resonance evaluation of atherosclerotic plaques in the human thoracic aorta: a comparison with transesophageal echocardiography. Circulation 101: 2503-2509.

32. Corti R (2006) Noninvasive imaging of atherosclerotic vessels by MRI for clinical assessment of the effectiveness of therapy. Pharmacol Ther 110: 57-70.

33. Freeman WD, Aguilar MI (2008) Stroke prevention in atrial fibrillation and other major cardiac sources of embolism. Neurol Clin 26: 1129-1160, x-xi.

34. Kronzon I, Tunick PA, Charney LH (2006) Echocardiography as a tool in the evaluation of conditions with a high likelihood of cardiogenic embolism. Isr Med Assoc J 8: 768-772.

35. Kim RJ, Fieno DS, Parrish TB, Harris K, Chen EL, et al. (1999) Relationship of MRI delayed contrast enhancement to irreversible injury, infarct age, and contractile function. Circulation 100: 1992-2002.

36. Kim RJ, Wu E, Rafael A, Chen EL, Parker MA, et al. (2000) The use of contrastenhanced magnetic resonance imaging to identify reversible myocardial dysfunction. N Engl J Med 343: 1445-1453.

37. Selvanayagam JB, Spyrou N, Francis JM, Neubauer S (2004) Resolution of ventricular thrombus identified by contrast enhanced cardiac MRI. Int J Cardiovasc Imaging 20: 369-370.

38. Ohyama H, Hosomi N, Takahashi T, Mizushige K, Osaka K, et al. (2003) Comparison of magnetic resonance imaging and transesophageal echocardiography in detection of thrombus in the left atrial appendage. Stroke 34: 2436-2439.

39. Dey D, Slomka P, Chien D, Fieno D, Abidov A, et al. (2006) Direct quantitative in vivo comparison of calcified atherosclerotic plaque on vascular MRI and CT by multimodality image registration. J Magn Reson Imaging 23: 345-354.

40. Mohrs OK, Petersen SE, Erkapic D, Rubel C, Schräder R, et al. (2005) Diagnosis of patent foramen ovale using contrast-enhanced dynamic MRI: a pilot study. AJR Am J Roentgenol 184: 234-240.

41. Rustemli A, Bhatti TK, Wolff SD (2007) Evaluating cardiac sources of embolic stroke with MRI. Echocardiography 24: 301-308.

42. Kerwin WS, Canton G (2009) Advanced techniques for MRI of atherosclerotic plaque. Top Magn Reson Imaging 20: 217-225. 